Pacific Journal of Mathematics

GAP SEQUENCES AT A SINGULARITY

September 1991 


\title{
GAP SEQUENCES AT A SINGULARITY
}

\author{
R. F. LAX AND CARL WIDLAND
}

The notion of Weierstrass gap sequence is extended to singular points on an integral projective Gorenstein curve. There are many gap sequences at such a point and the nongaps need not form a semigroup.

1. Let $X$ denote an integral, projective Gorenstein curve of arithmetic genus $g \geq 1$ defined over an algebraically closed field $k$. In previous articles $([6,11])$, we have defined Weierstrass points on such a curve if $k=\mathbf{C}$. We stated that the notions of Weierstrass gaps and nongaps did not seem to apply at a singular point $P$, since one is now interested in all 0-dimensional subschemes supported at $P$ and not the Weil divisors $n P$. Here, we present a generalization of gaps and nongaps to singular points by considering certain chains of ideals in the local ring at $P$.

Let $P$ be any closed point of $X$. Let $\mathscr{O}_{P}$ denote the local ring at $P$ and let $m_{P}$ denote the maximal ideal of $\mathscr{O}_{P}$. Then $\mathscr{O}_{P}$ is a one-dimensional Gorenstein domain. If $J$ is any nonzero ideal of $\mathscr{O}_{P}$, then the quotient ring $\mathscr{O}_{P} / J$ is a finite-dimensional vector space over $k$. We will let $\operatorname{col}(J)$ denote the colength of $J$, which is the dimension of the vector space $\mathscr{O}_{P} / J$.

(1.1) Definition. By a Weierstrass chain in $\mathscr{O}_{P}$, we will mean a chain $\mathscr{C}$ of ideals

$$
\mathscr{C}: m_{P}=J_{1} \supset J_{2} \supset \cdots \supset J_{2 g-1}
$$

such that $\operatorname{col}\left(J_{k}\right)=k$ for $k=1,2, \ldots, 2 g-1$.

First, we will see that every ideal of colength at most $2 g-1$ occurs in some Weierstrass chain.

(1.2) LeMmA. Let $J$ be a proper ideal of $\mathscr{O}_{P}$ with $\operatorname{col}(J)=n$.

1) There exists an ideal $J^{\prime}$ of $\mathscr{O}_{P}$ such that $J \subset J^{\prime}$ and $\operatorname{col}\left(J^{\prime}\right)=$ $n-1$.

2) There exists an ideal $J^{\prime \prime}$ of $\mathscr{O}_{P}$ such that $J \supset J^{\prime \prime}$ and $\operatorname{col}\left(J^{\prime \prime}\right)=$ $n+1$. 
Proof. We will prove (2). The proof of (1) is similar and is given in [11].

First, note that there are ideals of finite colength that are properly contained in $J$; for example, $m_{P} J$ cannot equal $(0)$, since $\mathscr{O}_{P}$ is a domain, and $m_{P} J$ cannot equal $J$ by Nakayama's Lemma. Let $J^{\prime \prime}$ be an ideal that is properly contained in $J$ and has minimum colength (necessarily at least $n+1$ ). Choose $x \in J$ with $x \notin J^{\prime \prime}$. It suffices to show that the image of $x$ generates the subspace $J / J^{\prime \prime}$ of the vector space $\mathscr{O}_{P} / J^{\prime \prime}$. By the choice of $J^{\prime \prime}$, we have $J^{\prime \prime}+(x)=J$. Suppose $y \in J$. Then there exists $z \in \mathscr{O}_{P}$ such that $y-x z \in J^{\prime \prime}$. Write $z=a+t$, where $t \in m_{P}$ and $a$ is either 0 or a unit in $\mathscr{O}_{P}$. We claim that $x t \in J^{\prime \prime}$. If $x t \notin J^{\prime \prime}$, then we would have $J^{\prime \prime}+(x t)=J$. Hence, there would exist $u \in \mathscr{O}_{P}$ such that $x-x t u=x(1-t u) \in J^{\prime \prime}$. But since $t \in m_{P}$, we have that $1-t u$ is a unit, so this would imply that $x \in J^{\prime \prime}$. Therefore, $x t \in J^{\prime \prime}$. Since $y-x z=y-a x-x t \in J^{\prime \prime}$, we may conclude that $y-a x \in J^{\prime \prime}$. Thus the image of $x$ in $J / J^{\prime \prime}$ generates $J / J^{\prime \prime}$ as a vector space over $k$.

(1.3) Corollary. Every ideal of $\mathscr{O}_{P}$ of colength at most $2 g-1$ occurs in some Weierstrass chain.

The next ingredient we need is a Riemann-Roch theorem for subschemes on a Gorenstein curve. If $Z$ is a proper closed subscheme of $X$ and if $\mathscr{I}$ is the sheaf of ideals defining $Z$, then the degree of $Z$, denoted $d(Z)$, is given by

$$
d(Z)=\sum_{Q \in \operatorname{Supp}(Z)} \operatorname{col}\left(\mathscr{I}_{Q}\right) .
$$

Let $\omega$ denote the sheaf of dualizing differentials on $X$. Since $X$ is Gorenstein, $\omega$ is locally free. With this notation, we have

(1.4) TheOREM. $\operatorname{dim}_{k} \operatorname{Hom}_{\mathscr{O}_{X}}\left(\mathscr{I}, \mathscr{O}_{X}\right)-\operatorname{dim}_{k} H^{0}\left(X, \mathscr{I} \otimes_{\mathscr{O}_{X}} \omega\right)=$ $d(Z)+1-g$.

Proof [11]. (Also, see [4].)

The elements of $\operatorname{Hom}_{\mathscr{O}_{X}}\left(\mathscr{I}, \mathscr{O}_{X}\right)$ may be identified with rational functions on $X$. Indeed, we have

$$
\operatorname{Hom}_{\mathscr{O}_{X}}\left(\mathscr{I}, \mathscr{O}_{X}\right)=\bigcap_{Q \in X} \operatorname{Hom}_{\mathscr{O}_{Q}}\left(\mathscr{I}_{Q}, \mathscr{O}_{Q}\right)
$$

and $\operatorname{Hom}_{\mathscr{O}_{Q}}\left(\mathscr{I}_{Q}, \mathscr{O}_{Q}\right)$ is the fractional ideal $\left(\mathscr{O}_{Q}: \mathscr{I}_{Q}\right)$ in the quotient field of $\mathscr{O}_{Q}$ for each $Q \in X$ (cf. [10], p. 37). Thus, if $Q$ is a smooth 
point and $\mathscr{I}$ is the invertible sheaf defining the divisor $n Q$, then $\operatorname{Hom}_{\mathscr{O}_{X}}\left(\mathscr{I}, \mathscr{O}_{X}\right)$ is just the space $H^{0}(X, \mathscr{O}(n Q))$.

If $J$ is a proper ideal of $\mathscr{O}_{P}$, let $\mathscr{I}(J)$ denote the sheaf of $\mathscr{O}_{X^{-}}$ ideals defined by $\mathscr{I}(J)_{P}=J$ and $\mathscr{I}(J)_{Q}=\mathscr{O}_{Q}$ for all $Q \neq P$. Put

$$
h(J)=\operatorname{dim}_{\mathbf{C}} \operatorname{Hom}_{\mathscr{O}_{X}}\left(\mathscr{I}(J), \mathscr{O}_{X}\right) .
$$

(1.5) LEMMA. (1) $h\left(m_{P}\right)=1$.

(2) If $J$ is a proper ideal of $\mathscr{O}_{P}$ and $\operatorname{col}(J)>2 g-2$, then $h(J)=$ $\operatorname{col}(J)+1-g$.

Proof. The first assertion is Lemma 1 of [11]. (This basically says that $\omega$ is base-point-free.) The second assertion follows from Theorem (1.4) if we can show that $H^{0}\left(X, \mathscr{I}(J) \otimes_{\mathscr{O}_{X}} \omega\right)=0$. Suppose $\sigma \in H^{0}\left(X, \mathscr{F}(J) \otimes_{\mathscr{O}_{X}} \omega\right)$. Then the image of $\sigma$ in $\omega_{P}$ lies in $J \omega_{P}$. If $\tau$ generates $\omega_{P}$, then locally at $P$ we may write $\sigma=f \tau$, with $f \in J$. If $\sigma \neq 0$, then we have

$$
\operatorname{ord}_{P} \sigma=\operatorname{ord}_{P} f=\operatorname{dim} \mathscr{O}_{P} /(f) \geq \operatorname{col}(J)>2 g-2 .
$$

But $\operatorname{ord}_{Q} \sigma \geq 0$ for all $Q \neq P$, so this would contradict the fact that the degree of a dualizing differential is $2 g-2$. Thus

$$
H^{0}\left(X, \mathscr{I}(J) \otimes_{\mathscr{O}_{X}} \omega\right)=0 \text {. }
$$

(1.6) Lemma. Suppose $J_{1}, J_{2}$ are nonzero ideals of $\mathscr{O}_{P}$ such that

$$
J_{1} \supset J_{2} \text { and } \operatorname{col}\left(J_{2}\right)=\operatorname{col}\left(J_{1}\right)+1 \text {. }
$$

Then either $h\left(J_{2}\right)=h\left(J_{1}\right)$ or $h\left(J_{2}\right)=h\left(J_{1}\right)+1$.

Proof. Applying the functor $\operatorname{Hom}_{\mathscr{O}_{X}}\left(, \mathscr{O}_{X}\right)$ to the exact sequence

$$
0 \rightarrow \mathscr{I}\left(J_{2}\right) \rightarrow \mathscr{I}\left(J_{1}\right) \rightarrow \mathscr{I}\left(J_{1}\right) / \mathscr{I}\left(J_{2}\right) \rightarrow 0,
$$

we obtain the exact sequence

$$
\begin{aligned}
0 & \rightarrow \operatorname{Hom}_{\mathscr{O}_{X}}\left(\mathscr{I}\left(J_{1}\right) / \mathscr{I}\left(J_{2}\right), \mathscr{O}_{X}\right) \rightarrow \operatorname{Hom}_{\mathscr{O}_{X}}\left(\mathscr{I}\left(J_{1}\right), \mathscr{O}_{X}\right) \\
& \rightarrow \operatorname{Hom}_{\mathscr{O}_{X}}\left(\mathscr{I}\left(J_{2}\right), \mathscr{O}_{X}\right) \rightarrow \operatorname{Ext}_{\mathscr{O}_{X}}^{1}\left(\mathscr{I}\left(J_{1}\right) / \mathscr{I}\left(J_{2}\right), \mathscr{O}_{X}\right) .
\end{aligned}
$$

The quotient sheaf $\mathscr{I}\left(J_{1}\right) / \mathscr{I}\left(J_{2}\right)$ is 0 at all points except $P$ and its stalk at $P$ is $J_{1} / J_{2} \cong k$. Since $\mathscr{O}_{P}$ is a one-dimensional CohenMacaulay ring, we have $\operatorname{Hom}_{\mathscr{O}_{X}}\left(\mathscr{I}\left(J_{1}\right) / \mathscr{I}\left(J_{2}\right), \mathscr{O}_{X}\right) \cong \operatorname{Hom}_{\mathscr{O}_{P}}\left(k, \mathscr{O}_{P}\right)$ $=0$ by $\left[9\right.$, Thm. 17.1]. And since $\mathscr{O}_{P}$ is a one-dimensional Gorenstein ring, we have $\operatorname{Ext}_{\mathscr{O}_{X}}^{1}\left(\mathscr{I}\left(J_{1}\right) / \mathscr{I}\left(J_{2}\right), \mathscr{O}_{X}\right) \cong \operatorname{Ext}_{\mathscr{O}_{P}}^{1}\left(k, \mathscr{O}_{P}\right) \cong k$ by [9, 
Thm. 18.1]. It now follows from $(*)$ that we have an inclusion

$$
\operatorname{Hom}_{\mathscr{O}_{X}}\left(\mathscr{I}\left(J_{2}\right), \mathscr{O}_{X}\right) / \operatorname{Hom}_{\mathscr{O}_{X}}\left(\mathscr{I}\left(J_{1}\right), \mathscr{O}_{X}\right) \hookrightarrow k .
$$

Thus, either $h\left(J_{2}\right)=h\left(J_{1}\right)$ or $h\left(J_{2}\right)=h\left(J_{1}\right)+1$.

The above lemmas are analogous to results one needs in the smooth case to prove the Weierstrass Gap Theorem. We can now give a generalization of this theorem to integral, projective Gorenstein curves.

(1.7) THEOREM (Weierstrass Gap Theorem for Gorenstein curves). Given any Weierstrass chain

$$
\mathscr{C}: m_{P}=J_{1} \supset J_{2} \supset \cdots \supset J_{2 g-1}
$$

in $\mathscr{O}_{P}$, there exist precisely $g$ integers

$$
1=\gamma_{1}<\gamma_{2}<\gamma_{3}<\cdots<\gamma_{g} \leq 2 g-1
$$

such that $h\left(J_{\gamma_{k}}\right)=h\left(J_{\gamma_{k}-1}\right)$ for $k=2,3, \ldots, g$.

Proof. From Lemma (1.5), we know that $h\left(m_{P}\right)=1$ and $h\left(J_{2 g-1}\right)$ $=g$. The Theorem now follows from Lemma (1.6).

(1.8) Definitions. We call the integers $\gamma_{k}, k=1,2, \ldots, g$, the Weierstrass gaps of the Weierstrass chain $\mathscr{C}$ and the sequence 1 , $\gamma_{2}, \ldots, \gamma_{g}$ will be called the Weierstrass gap sequence of $\mathscr{C}$. A nongap of the chain $\mathscr{C}$ is any nonnegative integer that is not a gap. The weight of the chain $\mathscr{C}$, denoted $w(\mathscr{C})$, is defined by

$$
w(\mathscr{C})=\sum_{k=1}^{g}\left(\gamma_{k}-k\right) \text {. }
$$

(1.9) Theorem. Assume $k=\mathrm{C}$ and $g>1$. If $P$ is a singular point of $X$, then there exists a Weierstrass chain in $\mathscr{O}_{P}$ of positive weight.

Proof. Under the given assumptions, we showed in [6] and [11] that every singular point of $X$ is a Weierstrass point and that a point $P$ is a Weierstrass point if and only if there exists an ideal $J$ of $\mathscr{O}_{P}$ such that $\operatorname{col}(J)=g$ and $h(J)>1$. By Corollary (1.3), there is a Weierstrass chain in $\mathscr{O}_{P}$ in which $J$ occurs. Since $h(J)>1$, there must be a nongap of this chain that is less than or equal to $g$. It follows that this chain has positive weight.

2. We will give two examples to illustrate phenomena that may occur in the singular case, but do not occur in the smooth case. First, there may be many different gap sequences possible at a singular point. 
Secondly, the nongaps of a chain at a singular point do not necessarily form a semigroup.

(2.1) EXAMPLE. Let $X$ be the projective plane curve over $\mathbf{C}$ defined by $y^{3} z=x^{4}$. Then $X$ is the image of $\mathbf{P}^{1}$ via the map

$$
(t, u) \mapsto\left(t^{3} u, t^{4}, u^{4}\right)
$$

and $X$ has arithmetic genus 3 . Let $P$ denote the point $(0,0,1)$, which is the only singularity of $X$. Let $\mathscr{O}$ denote the local ring at $P$ and let $\widetilde{\mathscr{O}}$ denote the normalization of $\mathscr{O}$. Then we have

$$
\mathscr{O}=\mathbf{C}+\mathbf{C} t^{3}+\mathbf{C} t^{4}+t^{6} \widetilde{O} .
$$

A basis for $H^{0}(X, \omega)$ is given by $d t / t^{2}, d t / t^{3}, d t / t^{6}$. According to the definition in [6] (or [11]), the point $P$ is a Weierstrass point of weight 22. To see this, note that the dualizing differential $\tau=d t / t^{6}$ generates $\omega_{P}$. We then write $d t / t^{6}=1 \tau, d t / t^{3}=t^{3} \tau, d t / t^{2}=t^{4} \tau$. In the notation of [6], we then have $F_{1,1}=1, F_{1,2}=t^{3}, F_{1,3}=t^{4}$ in a neighborhood of $P$. To compute the "wronskian" in [6], we must differentiate "with respect to $\tau$." For example, we have $d F_{1,2}=$ $3 t^{2} d t=3 t^{8} \tau$, and so $F_{2,2}=3 t^{8}$. The wronskian section $\rho$ in a neighborhood of $P$ is given by

$$
\rho=\left|\begin{array}{ccc}
1 & t^{3} & t^{4} \\
0 & 3 t^{8} & 4 t^{9} \\
0 & 24 t^{13} & 36 t^{14}
\end{array}\right|=12 t^{22}
$$

The point $(0,1,0)$ at infinity is a (smooth) Weierstrass point of weight 2 .

We will give examples of three Weierstrass chains in $\mathscr{O}$, each having a different gap sequence. For the first chain, consider

$$
\mathscr{C}_{1}:\left(t^{3}, t^{4}\right) \supset\left(t^{4}, t^{6}\right) \supset\left(t^{6}, t^{7}, t^{8}\right) \supset\left(t^{7}, t^{8}, t^{9}\right) \supset\left(t^{8}, t^{9}, t^{10}\right) .
$$

We then have the following table:

TABLE 1

\begin{tabular}{c|c|c|c|c}
\hline & & Basis of & Basis of & \\
& $\operatorname{col}(J)$ & $H^{0}(X, \mathscr{I}(J) \otimes \omega)$ & $\operatorname{Hom}\left(\mathscr{I}(J), \mathscr{O}_{X}\right)$ & $h(J)$ \\
\hline$\left(t^{3}, t^{4}\right)$ & 1 & $t^{3} \tau, t^{4} \tau$ & 1 & 1 \\
$\left(t^{4}, t^{6}\right)$ & 2 & $t^{4} \tau$ & 1 & 1 \\
$\left(t^{6}, t^{7}, t^{8}\right)$ & 3 & 0 & 1 & 1 \\
$\left(t^{7}, t^{8}, t^{9}\right)$ & 4 & 0 & $1, t^{-1}$ & 2 \\
$\left(t^{8}, t^{9}, t^{10}\right.$ & 5 & 0 & $1, t^{-1}, t^{-2}$ & 3 \\
\hline
\end{tabular}


Therefore, the gap sequence of this chain is $1,2,3$. This shows that there can be chains with zero weight at a singular point.

Now consider the chain

$$
\mathscr{C}_{2}:\left(t^{3}, t^{4}\right) \supset\left(t^{4}, t^{6}\right) \supset\left(t^{4}, t^{9}\right) \supset\left(t^{7}, t^{8}, t^{9}\right) \supset\left(t^{8}, t^{9}, t^{10}\right) .
$$

By proceeding as above, it can be seen that the gap sequence of this chain is $1,2,4$. (Note that if $J=\left(t^{4}, t^{9}\right)$, then a basis for $\operatorname{Hom}\left(\mathscr{I}(J), \mathscr{O}_{X}\right)$ is $1, t^{-1}$.) For a third chain, consider

$$
\mathscr{C}_{3}:\left(t^{3}, t^{4}\right) \supset\left(t^{4}, t^{6}\right) \supset\left(t^{4}, t^{9}\right) \supset\left(t^{4}\right) \supset\left(t^{7}, t^{8}\right) .
$$

Then the gap sequence of this chain is $1,2,5$. (Note that if $J=\left(t^{4}\right)$, then a basis for $\operatorname{Hom}\left(\mathscr{I}(J), \mathscr{O}_{X}\right)$ is $1, t^{-1}, t^{-4}$.) Therefore, all three possible gap sequences that can occur at points on smooth nonhyperelliptic curves of genus 3 occur as gap sequences of Weierstrass chains in the local ring of this singularity.

The example above of a weight 0 Weierstrass chain at a singularity can be generalized as follows.

(2.2) Proposition. Suppose $X$ is rational and has precisely one singularity $P$. Let $c_{P}$ denote the conductor of $\mathscr{O}_{P}$ in its integral closure $\widetilde{\mathscr{O}}_{P}$. Then any Weierstrass chain in $\mathscr{O}_{P}$ in which $c_{P}$ occurs has zero weight.

Proof. Since $\mathscr{O}_{P}$ is a one-dimensional Gorenstein ring, we have by [5] that $\operatorname{col}\left(c_{P}\right)=\operatorname{dim}_{k} \widetilde{\mathscr{O}}_{P} / \mathscr{O}_{P}$. Since $X$ is rational and $P$ is the only singularity, it follows that $\operatorname{col}\left(c_{P}\right)=g$, the arithmetic genus of $X$. It now suffices to show that $h\left(c_{P}\right)=1$, for then any Weierstrass chain in which $c_{P}$ occurs must have gap sequence $1,2, \ldots, g$.

We claim that $\operatorname{Hom}_{\mathscr{O}_{P}}\left(c_{P}, \mathscr{O}_{P}\right)=\widetilde{\mathscr{O}}_{P}$. From the definition of $c_{P}$, it is easy to see that $\widetilde{\mathscr{O}}_{P} \subseteq \operatorname{Hom}_{\mathscr{O}_{P}}\left(c_{P}, \mathscr{O}_{P}\right)$. Now, suppose $s$ generates $c_{P}$ in $\widetilde{\mathscr{O}}_{P}$ and let $f \in \operatorname{Hom}_{\mathscr{O}_{P}}\left(c_{P}, \mathscr{O}_{P}\right)$. Then $f s \widetilde{\mathscr{O}}_{P}=f c_{P} \subseteq \mathscr{O}_{P}$. Hence, by the definition of $c_{P}$, we have $f s \in c_{P}=s \widetilde{\mathscr{O}}_{P}$ and so $f \in$ $\widetilde{\mathscr{O}}_{P}$. Now, if $f \in \operatorname{Hom}_{\mathscr{O}_{X}}\left(\mathscr{I}\left(c_{P}\right), \mathscr{O}_{X}\right)$, then it follows that $f$ is a global regular function on the normalization of $X$, hence $f$ must be constant. Therefore, we have $h\left(c_{P}\right)=1$.

We note that $c_{P}=\left(t^{6}, t^{7}, t^{8}\right)$, as an ideal of $\mathscr{O}$, in Example (2.1).

(2.3) EXAMPLE. In this example, we show that the nongaps of a Weierstrass chain do not necessarily form an additive semigroup. Let 
$X^{\prime}$ be a nonsingular curve of genus 6 over $\mathbf{C}$ with a Weierstrass point $P^{\prime}$ such that the gap sequence at $P^{\prime}$ is $1,2,4,5,7,8$. (For compact Riemann surfaces, it was shown by Maclachlan [8] that every possible Weierstrass gap sequence with first nongap 3 actually occurs.) Let $t$ denote a local coordinate on $X^{\prime}$ centered at $P^{\prime}$. Let $\varphi_{1}, \varphi_{2}, \ldots, \varphi_{6}$ be the basis of the regular differentials on $X^{\prime}$ such that locally at $P^{\prime}$ we have

$$
\begin{gathered}
\varphi_{1}=d t, \quad \varphi_{2}=t d t, \quad \varphi_{3}=t^{3} d t, \quad \varphi_{4}=t^{4} d t, \\
\varphi_{5}=t^{6} d t, \quad \varphi_{6}=t^{7} d t .
\end{gathered}
$$

Now form a curve $X$ with arithmetic genus 7 by creating a cusp at $P^{\prime}$; i.e., $X$ will have a point $P$ such that $X \backslash\{P\} \cong X^{\prime} \backslash\left\{P^{\prime}\right\}$ and the local ring at $P$ will be

$$
\mathscr{O}_{P}=\mathbf{C}+t^{2} \mathscr{O}_{P^{\prime}}
$$

Then a basis for $H^{0}(X, \omega)$ is $\tau, \varphi_{1}, \varphi_{2}, \ldots, \varphi_{6}$, where locally at $P$ we have

$$
\begin{gathered}
\tau=d t / t^{2}, \quad \varphi_{1}=t^{2} \tau, \quad \varphi_{2}=t^{3} \tau, \quad \varphi_{3}=t^{5} \tau, \\
\varphi_{4}=t^{6} \tau, \quad \varphi_{5}=t^{8} \tau, \quad \varphi_{6}=t^{9} \tau .
\end{gathered}
$$

Consider the Weierstrass chain in $\mathscr{O}_{P}$ consisting of the ideals in the first column of Table 2 .

As can be seen from this table, the gap sequence of this Weierstrass chain is $1,2,3,5,6,8,9$. In particular, 4 is a nongap, but 8 is a

TABLE 2

\begin{tabular}{c|c|c|c}
\hline$J$ & $\operatorname{col}(J)$ & $H^{0}(X, \mathcal{I}(J) \otimes \omega)$ & $h(J)$ \\
\hline$\left(t^{2}, t^{3}\right)$ & 1 & $\varphi_{1}, \varphi_{2}, \varphi_{3}, \varphi_{4}, \varphi_{5}, \varphi_{6}$ & 1 \\
$\left(t^{3}, t^{4}\right)$ & 2 & $\varphi_{2}, \varphi_{3}, \varphi_{4}, \varphi_{5}, \varphi_{6}$ & 1 \\
$\left(t^{4}, t^{5}\right)$ & 3 & $\varphi_{3}, \varphi_{4}, \varphi_{5}, \varphi_{6}$ & 1 \\
$\left(t^{5}, t^{6}\right)$ & 4 & $\varphi_{3}, \varphi_{4}, \varphi_{5}, \varphi_{6}$ & 2 \\
$\left(t^{6}, t^{7}\right)$ & 5 & $\varphi_{4}, \varphi_{5}, \varphi_{6}$ & 2 \\
$\left(t^{7}, t^{8}\right)$ & 6 & $\varphi_{5}, \varphi_{6}$ & 2 \\
$\left(t^{8}, t^{9}\right)$ & 7 & $\varphi_{5}, \varphi_{6}$ & 3 \\
$\left(t^{9}, t^{10}\right)$ & 8 & $\varphi_{6}$ & 3 \\
$\left(t^{10}, t^{11}\right)$ & 9 & 0 & 3 \\
$\left(t^{11}, t^{12}\right)$ & 10 & 0 & 4 \\
$\left(t^{12}, t^{13}\right)$ & 11 & 0 & 5 \\
$\left(t^{13}, t^{14}\right)$ & 12 & 0 & 6 \\
$\left(t^{14}, t^{15}\right)$ & 13 & 0 & 7 \\
\hline
\end{tabular}


gap. We therefore have

(2.4) Proposition. The nongaps of a Weierstrass chain at a singular point do not necessarily form an additive semigroup.

We note that a possible reason for the failure of the semigroup property is that the colength of the product of two ideals in the local ring at a singularity need not equal the sum of the colengths of the two ideals.

3. On a more positive note, we will extend a well-known result in the smooth case by showing that if 2 is a nongap of a Weierstrass chain, then the curve is hyperelliptic. (We call an integral, projective, possibly singular, curve $X$ hyperelliptic if there exists a morphism $\varphi: X \rightarrow \mathbf{P}^{1}$ of degree 2 . Such a curve is necessarily Gorenstein [1].)

(3.1) LemmA. Suppose $J_{1} \supset J_{2} \supset \cdots \supset J_{2 g-1}$ is a Weierstrass chain in $\mathscr{O}_{P}$. If 2 is a nongap of this chain, then $J_{2}$ is a principal ideal.

Proof. We may suppose that $P$ is a singular point of $X$. Since 2 is a nongap, there exists a nonconstant function $f$ in $\operatorname{Hom}\left(\mathscr{I}\left(J_{2}\right), \mathscr{O}_{X}\right)$. Note that $f \in \mathscr{O}_{P^{\prime}}$ for all $P^{\prime} \neq P$. Let $\pi: \widetilde{X} \rightarrow X$ denote the normalization. If $f$ were in the local ring at every point of the fiber $\pi^{-1}(P)$, then $f$ would be in the local ring at every point of $\widetilde{X}$ and hence $f$ would be constant. Thus, since $f$ is nonconstant, there exists $Q \in \pi^{-1}(P)$ such that $\operatorname{ord}_{Q} f<0$.

Choose $\sigma \in H^{0}\left(X, \mathscr{I}\left(J_{2}\right) \otimes \omega\right)$ such that $\operatorname{ord}_{Q} \sigma$ is minimal (viewing $\sigma$ as a rational differential on $\tilde{X})$. Since $\sigma \in J_{2} \omega_{P}$ and $f \in$ $\operatorname{Hom}\left(\mathscr{I}\left(J_{2}\right), \mathscr{O}_{X}\right)$, we have that $f \sigma \in H^{0}(X, \omega)$. Since $\operatorname{ord}_{Q} f \sigma<$ $\operatorname{ord}_{Q} \sigma$, we must have $f \sigma \notin J_{2} \omega_{P}$. We claim that this implies that $f \sigma$ generates $\omega_{P}$. Indeed, by Theorem (1.4),

$$
\operatorname{dim} H^{0}\left(X, \mathscr{I}\left(J_{2}\right) \otimes \omega\right)=g-1,
$$

so we may choose a basis $\sigma_{2}, \ldots, \sigma_{g}$ of $H^{0}\left(X, \mathscr{I}\left(J_{2}\right) \otimes \omega\right)$ such that $f \sigma, \sigma_{2}, \ldots, \sigma_{g}$ is a basis of $H^{0}(X, \omega)$. Since $\sigma_{2}, \ldots, \sigma_{g}$ are in $m_{P} \omega_{P}$ and since $h\left(m_{P}\right)=1$, we have that $f \sigma \notin m_{P} \omega_{P}$. It follows that $f \sigma$ generates $\omega_{P}$.

Now write $\sigma=f_{1} f \sigma$ for some $f_{1} \in \mathscr{O}_{P}$. Note that $f_{1}$ is not a unit in $\mathscr{O}_{P}$ since $f \notin \mathscr{O}_{P}$. Now, given any $\bar{f} \in J_{2}$, we have $\bar{f}=$ $(f \bar{f}) f_{1}$, where $f \bar{f} \in \mathscr{O}_{P}$ since $f \in \operatorname{Hom}\left(\mathscr{I}\left(J_{2}\right), \mathscr{O}_{X}\right)$. It follows that $J_{2} \subseteq f_{1} \mathscr{O}_{P}$. But $J_{2}$ has colength 2 and the maximal ideal of $\mathscr{O}_{P}$ is not principal, so we conclude that $J_{2}=f_{1} \mathscr{O}_{P}$. 
We note that Example (2.1) shows that Lemma (3.1) fails if "2" is replaced by " 3 ".

(3.2) Theorem. Suppose $k=\mathrm{C}$. The following are equivalent:

(1) $X$ is hyperelliptic.

(2) At every Weierstrass point of $X$, there is a Weierstrass chain with 2 as a nongap.

(3) At some Weierstrass point of $X$, there is a Weierstrass chain with 2 as a nongap.

Proof. (1) $\Rightarrow(2)$ : Suppose $\varphi: X \rightarrow \mathbf{P}^{1}$ is a morphism of degree 2 and let $P$ be a singular point of $X$. By composing $\varphi$ with an automorphism of $\mathbf{P}^{1}$, we may assume that $\varphi(P)=0$. Let $\theta: \mathbf{C}(T) \rightarrow$ $K(X)$ be the field homomorphism associated to $\varphi$ and suppose $f=$ $\theta(T)$. Then $f \in m_{P}$. Since $P$ is singular, $\operatorname{ord}_{P} f \geq 2$, and since $\varphi$ has degree 2 , we conclude that $\operatorname{ord}_{P} f=2$. It follows that $f$ has no other zeros on $X$ and so $\varphi^{-1}(0)=\{P\}$. Then the ideal $J$ generated by $f$ in $\mathscr{O}_{P}$ has colength 2 and $f^{-1}$ is a nonconstant element in $\operatorname{Hom}_{\mathscr{O}_{X}}\left(\mathscr{I}(J), \mathscr{O}_{X}\right)$. Thus 2 is a nongap of any Weierstrass chain in $\mathscr{O}_{P}$ that contains $J$.

Now we need to show that 2 is a nongap at every smooth Weierstrass point of $X$. Let $\pi: \widetilde{X} \rightarrow X$ denote the normalization of $X$ and let $\tilde{g}$ denote the genus of $\tilde{X}$. Then $\varphi \circ \pi: \widetilde{X} \rightarrow \mathbf{P}^{1}$ has degree 2 . Let $Q_{1}, \ldots, Q_{2 \tilde{g}+2}$ be the ramification points of $\varphi \circ \pi$. (These are exactly the Weierstrass points of $\tilde{X}$ if $\tilde{g}>1$.) Put $P_{i}=\pi\left(Q_{i}\right)$, $i=1, \ldots, 2 \tilde{g}+2$. Some of the $P_{i}$ 's may be smooth points and some may be (unibranch) singularities. If $P_{i}$ is a smooth point of $X$, then the Weierstrass weight of $P_{i}$ will be $(g-1) g / 2$, just as in the classical case. We need to see that the smooth points among the $P_{i}$ 's are precisely all the smooth Weierstrass points of $X$ and to see this, we need to compute the Weierstrass weight (as in [6] or [11]) of the singular points among the $P_{i}$ 's.

So, suppose $P$ is a unibranch singularity of $X$ such that $\pi^{-1}(P)$ is a branch point of $\varphi \circ \pi$. Let $\psi: X^{\prime} \rightarrow X$ denote the partial normalization of $X$ at $P$ and let $Q$ denote $\psi^{-1}(P)$. The genus of $X^{\prime}$ is $g^{\prime}=g-\delta_{P}$, where $\delta_{P}=\operatorname{dim} \mathscr{O}_{Q} / \mathscr{O}_{P}$. Let $f \in K(X)=K\left(X^{\prime}\right)$ be a rational function with order 2 at $P$ (and at $Q$ ) and no other zeros. We may choose a basis $\sigma_{1}, \sigma_{2}, \ldots, \sigma_{g^{\prime}}$ of $H^{0}\left(X^{\prime}, \omega_{X^{\prime}}\right)$ and a local coordinate $t$ on $X^{\prime}$ centered at $Q$ such that locally at $Q$ we have $\sigma_{j}=f^{j-1} d t$ for $j=1,2, \ldots, g^{\prime}$. Now, it is not hard to see that the local ring at $P$ is of the form

$$
\mathscr{O}_{P}=\mathbf{C}+f \mathbf{C}+f^{2} \mathbf{C}+\cdots+f^{\delta_{P}-1} \mathbf{C}+f^{\delta_{P}} \mathscr{O}_{Q} .
$$


A basis of $H^{0}(X, \omega)$ is then $\sigma_{1}, \ldots, \sigma_{g^{\prime}}, \sigma_{g^{\prime}+1}, \sigma_{g^{\prime}+2}, \ldots, \sigma_{g}$, where locally at $P$ we have

$$
\sigma_{k}=\frac{d t}{f^{k-g^{\prime}}} \text { for } k=g^{\prime}+1, \ldots, g .
$$

Now, $\omega_{P}$ is generated by $\tau=\sigma_{g}=d t / f^{\delta_{P}}$. To compute the Weierstrass weight of $P$ we need to write each of the dualizing differentials in the above basis as a rational function times $\tau$ and then we need to form the wronskian by differentiating "with respect to $\tau$." Reordering the basis above as $\sigma_{g}, \ldots, \sigma_{g^{\prime}+1}, \sigma_{1}, \ldots, \sigma_{g^{\prime}}$, we have that locally at $P$ we may write these differentials as

$$
\tau, f \tau, f^{2} \tau, \ldots, f^{g-1} \tau .
$$

It is then not hard to see, as in the proof of Proposition 3 in [6], that $W(P)$, the Weierstrass weight of $P$, is $W(P)=\delta_{P}(g-1) g+$ $\operatorname{ord}_{Q} W_{t}\left(1, f, f^{2}, \ldots, f^{g-1}\right)$, where $W_{t}$ is the usual wronskian of rational functions obtained by differentiating with respect to $t$. Since $f$ has order 2 at $Q$, we have

$$
\begin{aligned}
\operatorname{ord}_{Q} & W_{t}\left(1, f, f^{2}, \ldots, f^{g-1}\right) \\
& =(0-0)+(2-1)+(4-2)+\cdots+(2(g-1)-g) \\
& =1+2+3+\cdots+g-1=(g-1) g / 2 .
\end{aligned}
$$

Therefore, the Weierstrass weight of $P$ is $\left(\delta_{P}+1 / 2\right)(g-1) g$.

Now, suppose $P_{1}, P_{2}, \ldots, P_{r}$ are the unibranch singularities of $X$ and $P_{r+1}, P_{r+2}, \ldots, P_{2 \tilde{g}+2}$ are smooth points of $X$ such that $\pi^{-1}\left(P_{i}\right)$ is a branch point of $\varphi \circ \pi$ for $i=1,2, \ldots, 2 \tilde{g}+2$. $X$ may have other singularities (necessarily double points), so let $R_{1}, \ldots, R_{s}$ be the other singular points of $X$. From [6], we have that for $j=$ $1, \ldots, s, W\left(R_{j}\right) \geq \delta_{R_{j}}(g-1) g$, where $\delta_{R_{j}}=\operatorname{dim} \widetilde{\mathscr{O}}_{R_{j}} / \mathscr{O}_{R_{j}}$ and $\widetilde{\mathscr{O}}_{R_{j}}$ is the normalization of $\mathscr{O}_{R_{j}}$. The sum of the Weierstrass weights of all points of $X$ is $g^{3}-g$ and the sum of the weights of the $P_{i}$ 's and $R_{j}$ 's is at least

$$
\begin{aligned}
& \sum_{i=1}^{r}\left(\delta_{P_{\imath}}+1 / 2\right)(g-1) g+(2 \tilde{g}+2-r)(g-1) g / 2+\sum_{j=1}^{s} \delta_{R_{j}}(g-1) g \\
& \quad=(g-1) g\left(\tilde{g}+1+\sum_{i=1}^{r} \delta_{P_{\imath}}+\sum_{j=1}^{s} \delta_{R_{j}}\right) \\
& \quad=(g-1) g(g+1)=g^{3}-g,
\end{aligned}
$$


since $\tilde{g}=g-\sum_{i=1}^{r} \delta_{P_{i}}-\sum_{j=1}^{s} \delta_{R_{j}}$. Therefore, there are no other smooth Weierstrass points of $X$.

$(2) \Rightarrow(3)$ : This is obvious. (3) $\Rightarrow(1)$ : Suppose $P$ is a Weierstrass point of $X$ such that 2 is a nongap of a Weierstrass chain at $P$. If $P$ is smooth, then (1) follows as in the classical case. If $P$ is a singular point, then by Lemma (3.1) there exists a rational function $f \in K(X)$ such that $f^{-1} \in \mathscr{O}_{P}$, ord $f^{-1}=2$, and $h\left(f^{-1} \mathscr{O}_{P}\right)=2$. It then follows from Proposition 4 of [11] that there exists a morphism $\varphi: X \rightarrow \mathbf{P}^{1}$ of degree 2 .

(3.3) COROLlARY. Every integral projective Gorenstein curve of arithmetic genus 2 over $\mathbf{C}$ is hyperelliptic.

Proof. Such a curve has Weierstrass points and the first nongap of any positive weight Weierstrass chain at a point on a curve of arithmetic genus 2 must be 2 .

4. The main open problem here is to determine a relationship between the Weierstrass weight of a singular point, as defined in [6] or [11], and the weights of the various Weierstrass chains in the local ring of that singularity. This amounts to relating the vanishing orders at a point of a basis of dualizing differentials with the Weierstrass nongaps at that point. We note that in Example (2.1), where the Weierstrass weight of $P$ is 22 , if one considers only ideals generated by monomials in $t$, then there are 14 Weierstrass chains of weight 0 , five Weierstrass chains of weight 1 each, and one Weierstrass chain of weight 2 . At a cusp on a rational curve with $g$ simple cusps, if one considers only ideals generated by monomials in a uniformizing parameter at the smooth point on the normalization lying over the cusp, then there are $F_{g-1} F_{g}$ weight 1 Weierstrass chains, where $F_{n}$ denotes the $n$th term in the Fibonacci sequence. This number is bigger than the Weierstrass weight of such a cusp for $g \geq 6$, so it appears that not all the Weierstrass chains contribute to the Weierstrass weight.

Weierstrass chains should also be related to the following question. One knows that as a family $\mathscr{X} \rightarrow T$ of smooth curves degenerates to a singular, integral Gorenstein curve, many of the Weierstrass points on the smooth curves tend to the singularities and the Weierstrass weight of a singularity is the sum of the Weierstrass weights of converging points on nearby smooth curves (see [7]). But what exactly happens in the limit to the gap sequences at the Weierstrass points on the smooth curves? Which Weierstrass chains occur as limits of the divisors $P_{t}, 2 P_{t}, 3 P_{t}, \ldots,(2 g-1) P_{t}$, where $\left\{P_{t}\right\}_{t \in T}$ is a family of 
points on the smooth curves converging to a singular point of the limit curve? This should be related to work of Eisenbud and Harris [2, 3].

\section{REFERENCES}

[1] F. Catanese, Pluricanonical Gorenstein curves, in Enumerative geometry and classical algebraic geometry (Nice, 1981), pp. 51-95, Progress in Math. 24, Birkhäuser, Boston, 1982.

[2] D. Eisenbud and J. Harris, When ramification points meet, Invent. Math., 87 (1987), 485-493.

[3] __, Existence, decomposition, and limits of certain Weierstrass points, Invent. Math., 87 (1987), 495-515.

[4] R. Hartshorne, Generalized divisors on Gorenstein curves and a theorem of Noether, J. Math. Kyoto Univ., 26 (1986), 375-386.

[5] E. Kunz, The value-semigroup of a one-dimensional Gorenstein ring, Proc. Amer. Math. Soc., 25 (1970), 748-751.

[6] R. F. Lax, On the distribution of Weierstrass points on singular curves, Israel J. Math., 57 (1987), 107-115.

[7] _- Weierstrass weight and degenerations, Proc. Amer. Math. Soc., 101 (1987), $8-10$.

[8] C. Maclachlan, Weierstrass points on compact Riemann surfaces, J. London Math. Soc. (2), 3 (1971), 722-724.

[9] H. Matsumura, Commutative Ring Theory, Cambridge University Press, Cambridge, 1986.

[10] E. Matlis, Torsion-free Modules, Univ. of Chicago Press, Chicago, 1972.

[11] C. Widland and R. F. Lax, Weierstrass points on Gorenstein curves, Pacific J. Math., 142 (1990), 197-208.

Received October 4, 1989 and in revised form January 8, 1990. The second author was supported by an Indiana University at Kokomo Summer Fellowship.

LOUISIANA STATE UNIVERSITY

BATON ROUGE, LA 70803

AND

INDIANA UNIVERSITY AT KOKOMO

KоKомо, IN 46901 


\section{PACIFIC JOURNAL OF MATHEMATICS EDITORS}

\author{
V. S. VARADARAJAN \\ (Managing Editor) \\ -University of California \\ Los Angeles, CA 90024-1555-05 \\ Herbert Clemens \\ University of Utah \\ Salt Lake City, UT 84112 \\ THOMAS ENRIGHT \\ University of California, San Diego \\ La Jolla, CA 92093
}

R. FINN

Stanford University

Stanford, CA 94305

Hermann FlaschKa

University of Arizona

Tucson, AZ 85721

VAUGHAN F. R. JONES

University of California

Berkeley, CA 94720

SteVen KerCKhoff

Stanford University

Stanford, CA 94305
C. C. MOORE

University of California

Berkeley, CA 94720

Martin ScharlemanN

University of California

Santa Barbara, CA 93106

\section{HAROLD STARK}

University of California, San Diego

La Jolla, CA 92093

\section{ASSOCIATE EDITORS}

\begin{tabular}{|c|c|c|c|c|}
\hline R. Arens & $\begin{array}{l}\text { E. F. BECKENBACH } \\
(1906-1982)\end{array}$ & Neumann & $\begin{array}{l}\text { F. WolF } \\
(1904-1989)\end{array}$ & K. Yoshida \\
\hline \multicolumn{5}{|c|}{ SUPPORTING INSTITUTIONS } \\
\hline \multicolumn{2}{|c|}{ UNIVERSITY OF ARIZONA } & \multicolumn{3}{|c|}{ UNIVERSITY OF OREGON } \\
\hline \multirow{2}{*}{\multicolumn{2}{|c|}{$\begin{array}{l}\text { UNIVERSITY OF BRITISH COLUMBIA } \\
\text { CALIFORNIA INSTITUTE OF TECHNOLOGY }\end{array}$}} & \multicolumn{3}{|c|}{ UNIVERSITY OF SOUTHERN CALIFORNIA } \\
\hline & & \multicolumn{3}{|c|}{ STANFORD UNIVERSITY } \\
\hline CALIFORNIA INSTITUTE OF TECHNOLOGY & UNIVERSITY OF CALIFORNIA & \multicolumn{3}{|c|}{ UNIVERSITY OF HAWAII } \\
\hline \multicolumn{2}{|c|}{ MONTANA STATE UNIVERSITY } & \multicolumn{3}{|c|}{ UNIVERSITY OF TOKYO } \\
\hline \multicolumn{2}{|c|}{ UNIVERSITY OF NEVADA, RENO } & \multicolumn{3}{|c|}{ UNIVERSITY OF UTAH } \\
\hline \multicolumn{2}{|c|}{ NEW MEXICO STATE UNIVERSITY } & \multicolumn{3}{|c|}{ WASHINGTON STATE UNIVERSITY } \\
\hline OREGON STA & UNIVERSITY & UNIVERS & OF WASHING & \\
\hline
\end{tabular}




\section{Pacific Journal of Mathematics}

Vol. 150, No. $1 \quad$ September, 1991

Jan Aarts, Charles Lemuel Hagopian and Lex Gerard Oversteegen, The orientability of matchbox manifolds $\ldots \ldots \ldots \ldots \ldots \ldots \ldots \ldots \ldots \ldots \ldots \ldots$

Roger W. Barnard, Carl Hanson Fitzgerald and Sheng Gong, The growth and $1 / 4$-theorems for starlike mappings in $\mathbf{C}^{n} \ldots \ldots \ldots \ldots \ldots \ldots \ldots$

Allan Berele, Trace rings for verbally prime algebras $\ldots \ldots \ldots \ldots \ldots \ldots \ldots 23$

Quo-Shin Chi, Curvature characterization and classification of rank-one

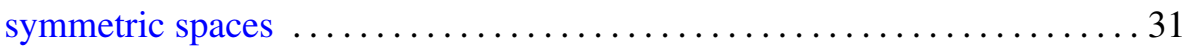

Harold Gerard Donnelly and Jeffrey Marc Lee, Domains in Riemannian

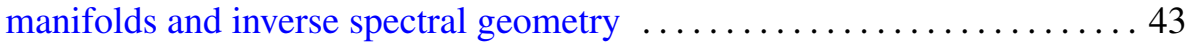

Clifford John Earle, Jr. and Patricia Lilaine Sipe, Families of Riemann

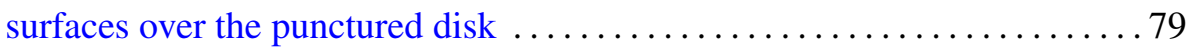

Mark P. Hughes, Dihedral group actions on homotopy complex projective

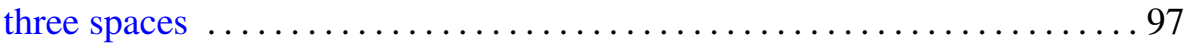

Robert F. Lax and Carl Widland, Gap sequences at a singularity ........111

Takahiko Nakazi, Bounded Hankel forms with weighted norms and lifting

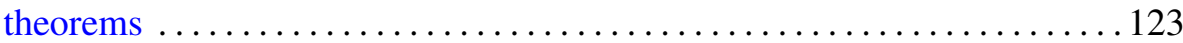

Douglas Murray Pickrell, Mackey analysis of infinite classical motion groups

Martha Rzedowski-Calderón and Gabriel Daniel Villa-Salvador,

Automorphisms of congruence function fields 167

Peter N-S Wong, Equivariant Nielsen fixed point theory for $G$-maps 